\title{
New insights into fragile $X$ syndrome: from molecules to neurobehaviors
}

\author{
Peng Jin and Stephen T. Warren \\ Department of Human Genetics, Emory University School of Medicine, 615 Michael Street GA 30322, Atlanta, USA
}

\begin{abstract}
Fragile $X$ syndrome - a common form of inherited mental retardation - is caused by the loss of the fragile $X$ mental retardation 1 protein (FMRP). FMRP is an RNA-binding protein which forms a messenger ribonucleoprotein (mRNP) complex that associates with translating polyribosomes. It has been proposed that FMRP is involved in synaptic plasticity through the regulation of mRNA transportation and translation. Recent advances in the identification of the mRNA ligands that are bound by FMRP, the RNA sequence and structure required for FMRP-RNA interaction, and the physiological consequences of FMRP deficiency in the brain are important steps towards understanding the molecular pathogenesis of fragile $X$ syndrome, and learning and memory in general.
\end{abstract}

Fragile $\mathrm{X}$ syndrome is the most common form of inherited mental retardation, with the estimated prevalence of one in 4000 males and one in 8000 females. In addition to cognitive deficits, the phenotype of fragile $\mathrm{X}$ syndrome includes mildly abnormal facial features (a prominent jaw, high forehead and large ears), MACROORCHIDISM (see Glossary) in postpubescent males and subtle connective tissue abnormalities [1]. Many patients also manifest attention-deficit hyperactivity disorder and autistic-like behavior. As one of the first identified human disorders caused by trinucleotide repeat expansion, fragile $\mathrm{X}$ syndrome is the result of a massive CGG trinucleotide repeat expansion within the gene fragile $\mathrm{X}$ mental retardation 1 (FMR1). FMR1 is a highly conserved gene that consists of 17 exons, and spans $\sim 38$ kilobases $(\mathrm{kb})$. Within the 4.4-kb FMR1 transcript, a CGG trinucleotide repeat is located in the $5^{\prime}$ untranslated region (UTR) [2]. Among normal individuals, this CGG repeat is highly polymorphic in length and content, often punctuated by AGG interruptions. The normal repeat size ranges from 7 to $\sim 54$, with 30 repeats found in the most common allele. In most affected individuals, CGG repeats are massively expanded over 230 repeats (full mutation) and become abnormally hypermethylated, which results in the transcriptional silencing of FMR1 [1]. Identification of other mutations in FMR1 (e.g. deletions and a point mutation among patients with the typical phenotype, but without FRAGILE SITE expression) has confirmed that the FMR1 gene is the only gene involved in the pathogenesis of fragile $\mathrm{X}$ syndrome and the loss of FMR1 product - fragile $\mathrm{X}$ mental retardation protein (FMRP) - causes fragile $\mathrm{X}$ syndrome [1]. A mouse model of fragile $\mathrm{X}$ syndrome was created using gene targeting; the Fmr1-knockout mice exhibit macroorchidism and subtle deficits in learning and memory, which mimic the human phenotype [3].

In this review, we discuss the recent progress in understanding the biological functions of FMRP and the physiological consequences of its absence that lead to mental retardation.

\section{Features of FMRP}

FMRP is widely expressed in fetal and adult tissues, with the most abundant expression in brain and testes [4]. FMRP, and its autosomal paralogs the fragile-X-related protein FXR1P and FXR2P, consists of a small family of RNA-binding proteins (fragile X-related gene family) $[5,6]$. These proteins share $>60 \%$ amino acid identity and contain two types of RNA-binding motifs: two ribonucleoprotein K homology domains (KH domains) and a cluster of arginine and glycine residues (RGG box). Owing to their similarities, it has been postulated that FXR1P and FXR2P can partially compensate for the loss of FMRP, although expression levels of both FXR1P and FXR2P are not altered in the cells from fragile X patients or the Fmr1knockout mice [7]. The fragile X-related gene family has been well conserved during evolution. The orthologs of the genes encoding FMRP, FXR1P and FXR2P are all identified in mouse, chicken and Xenopus. However, Drosophila contains only a single gene of the fragile Xrelated gene family, $d f m r 1$ (http://flybase.bio.indiana.edu; $d f m r 1$ has higher overall similarity to human FXR2 and also is called $d f x r$ for this reason) $[8,9]$.

\footnotetext{
Glossary

Dendritic spine dysgenesis: misregulation of dendritic spine formation during the development of nervous system.

Fragile site: specific chromosomal regions that form gaps, breaks and rearrangements when cells are cultured under conditions that inhibit DNA replication.

G-quartet: hydrogen-bonded structures formed from four guanosine residues in a square-planar array that are stabilized preferentially by $\mathrm{K}^{+}$and disrupted by the presence of $\mathrm{Li}^{+}$.

Macroorchidism: the condition (as in fragile $\mathrm{X}$ syndrome) of having enlarged testicles.

RNA homopolymer: large molecules consisting of $>$ fifty repeated ribonucleotides.

Synaptic boutons: the smallest 'unit synapses', which release approximately one vesicle per stimulus.
} 
As an RNA-binding protein, FMRP has been shown to bind to RNA HOMOPOLYMERS as well as a subset of brain transcripts in vitro [10]. FMRP is associated with actively translating polyribosomes in an RNA-dependent manner via messenger ribonucleoprotein (mRNP) particles [7]. A missense mutation in the second $\mathrm{KH}$ domain of FMRP (I304N), which results in severe mental retardation, prevents this polyribosome association, suggesting that the association of FMRP with polyribosomes is functionally important [7].

Although FMRP is predominantly localized in the cytoplasm, both a functional nuclear localization signal (NLS) and a nuclear export signal (NES) have been identified within FMRP, suggesting that FMRP might shuttle between the nucleus and cytoplasm [11]. It has been demonstrated that FMRP exists in the nucleus, and has been observed, using electron microscopy, in transit through the nuclear pore [12]. Pathological studies from the brains of both human patients with fragile X syndrome and from Fmr1-knockout mice have shown abnormal dendritic spines, which implicates a role for FMRP in synaptic plasticity [13-15]. Indeed, FMRP has been observed associated with polyribosomes in the synapses of wild-type neurons [12].

These biochemical and pathological studies have led to a proposed model of FMRP neuronal function (Fig. 1). FMRP is transported into the nucleus of neurons via its nuclear localization signal and, there, assembles into a mRNP complex thereby interacting with specific RNA transcripts and other proteins. Subsequently, the FMRP-mRNP complex is transported out of the nucleus via the NES of FMRP. Alternatively, FMRP might bind to RNA and associate with the mRNP complex in the cytoplasm. Once in the cytoplasm, the FMRP-mRNP complex can either associate with ribosomes in the cell body, or it can be transported into dendrites and perhaps regulate local protein synthesis of specific mRNAs in response to synaptic stimulation signals. When FMRP is absent, the mRNAs normally associated with FMRP-mRNP complexes might be translationally misregulated, which would lead to impaired synaptic plasticity and cognitive deficit. However, in this model, two key pieces of data had been missing until recently: the identity of mRNA ligands specifically regulated by FMRP, and physiological consequences associated with the loss of FMRP. Using biochemical, genetic, genomic and electrophysiological approaches together with different model systems, several recent studies have provided new insights into the molecular basis of fragile $\mathrm{X}$ syndrome, and could lead to the development of effective intervention for this disease.

\section{FMRP-mRNP complex: protein components, mRNA ligands and its role in translation}

The majority of cytoplasmic FMRP is a component of a large mRNP complex, which contains multiple proteins and FMRP and/or mRNA ligands. Using a murine cell culture system expressing epitope-tagged FMRP, several protein components of the FMRP-mRNP complex that are co-immunoprecipitated with tagged FMRP have been identified, including FXR1P, FXR2P, nucleolin and YB1/p50 [16,17]. Also through co-immunoprecipitation, using an antibody against Pur $\alpha$, FMRP was found to be part of an mRNP complex containing Pur $\alpha$ and $m$ Staufen proteins in mouse brain [18]. Pur proteins are predominantly expressed in neuronal cytoplasm and are translocated into dendrites [19]. mStaufen is involved in the

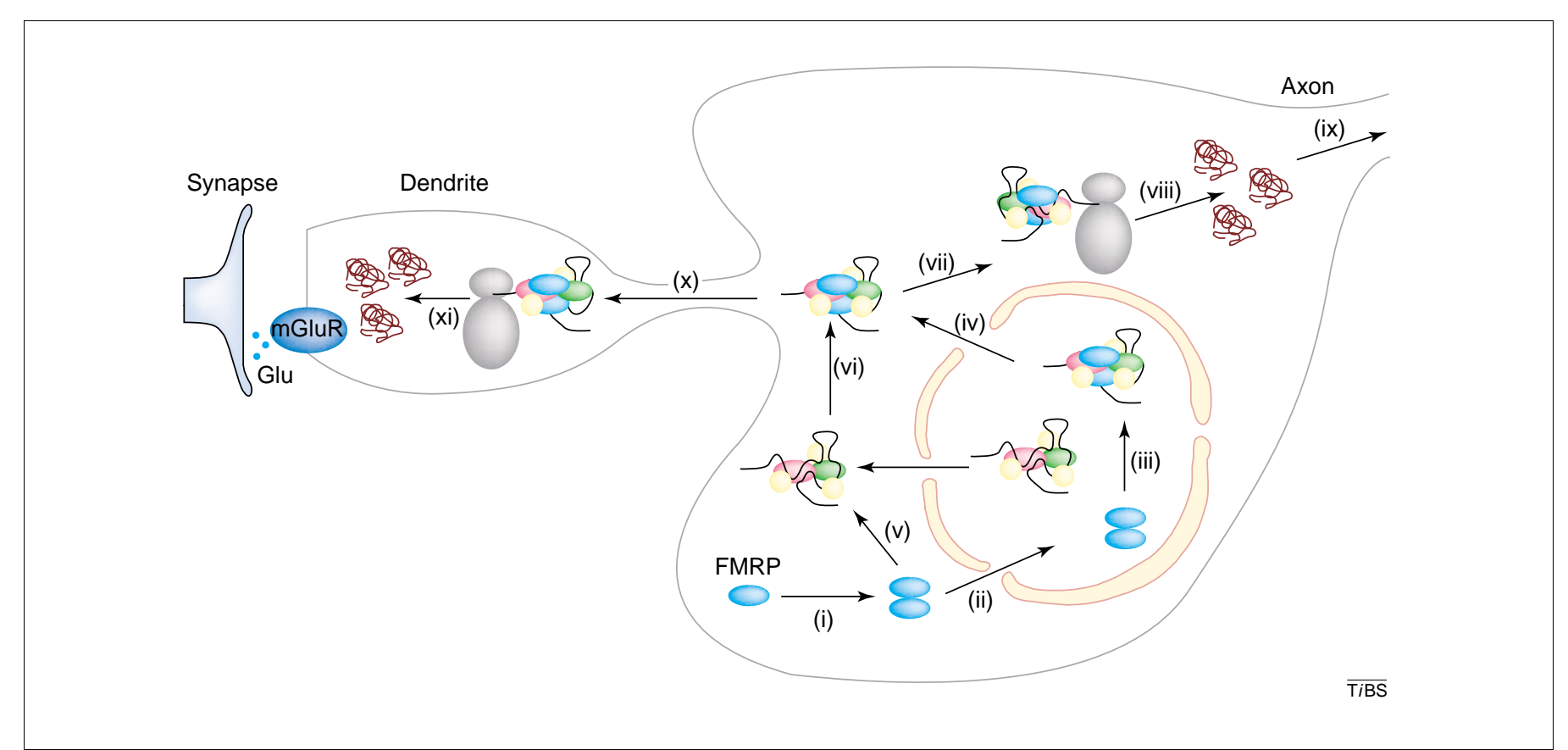

Fig. 1. Model of fragile $X$ mental retardation protein (FMRP) function in the neuron. FMRP is dimerized in the cytoplasm (i), and enters the nucleus of neurons via its nuclear localization signal (ii). FMRP then assembles into a complex with messenger ribonucleoprotein (mRNP), and interacts with specific RNA transcripts and other proteins (iii). The FMRP-mRNP complex is subsequently transported out of the nucleus via the nuclear export signal of FMRP (iv). Alternatively, FMRP might bind to RNA and associate with the mRNP complex in the cytoplasm $(v, v i)$. Once in the cytoplasm, the FMRP-mRNP complex can associate with ribosomes in the cell body (vii) to produce the proteins (viii), some of which might be important for axon guidance (ix). Alternatively, the FMRP-mRNP complex itself can be transported into dendrites ( $x$ ) and perhaps regulate local protein synthesis of specific RNAs in response to synaptic stimulation signals such as metabotropic glutamate receptor (mGluR) activation (xi). 
delivery of RNA to dendrites, and has been suggested to play a role in the transport of neuronal RNA granules, which might form a link between RNA localization and activity-dependent translation within dendrites [20,21]. In addition, this type of $\mathrm{mRNP}$ appears to reside on rough endoplasmic reticulum ( $r E R$ ) that is equipped with a kinesin motor [18], which might provide the basis for the transportation of the FMRP-mRNP complex into the dendrites. In fact, using a newly developed PC12 cell line with an inducible expression of the FMRP-green fluorescent protein (GFP) fusion protein, the trafficking of FMRP-GFP granules into the neurites of living cells has been demonstrated [22]. Motile FMRP-GFP granules display two types of movement: oscillatory (bidirectional) and unidirectional anterograde. The movement of FMRPGFP granules is microtubule-dependent [22]. In addition, using a yeast two-hybrid screen, several FMRP-interacting proteins [nuclear FMRP-interacting protein 1 (NUFIP1), cytoplasmic FMRP-interacting protein 1 (CYFIP1) (KIAA0068, p140Sra-1) and CYFIP2 (PIR121)] have been identified [23,24]. However, whether they are part of the FMRP-mRNP complex remains to be determined. Interestingly, despite the high similarity between FMRP and FXR1P or FXR2P, NUFIP1 and CYFIP1 only interact with FMRP. This suggests that these two proteins might be involved in the transportation and/or translational control of a subset of mRNAs specifically regulated by FMRP [24].

Although FMRP has been shown to bind to $4 \%$ of brain mRNAs in vitro, the identity of the mRNA ligands specifically regulated by FMRP has remained elusive [10]. Several groups have taken different approaches to identify the mRNAs that are specifically bound by FMRP and the structure required for FMRP-RNA interaction [25-27]. To identify the in vivo mRNA targets for FMRP, our group used microarrays to perform a genome-wide search [25]. The mRNA was co-immunoprecipitated with the FMRP - mRNP complex from mouse brain and used for microarray analysis. By comparing the input RNA used for immunoprecipitation and the immunoprecipitated RNA from the Fmr1-knockout-mouse brain, we have acheived en masse identification of the mRNAs associated with the FMRP-mRNP complex. As FMRP is involved in translation and has been found associated with polyribosomes, in parallel studies we compared the mRNA profiles in polyribosomal fractions of normal and fragile X cells. We have identified the mRNAs that display an abnormal polyribosome profile in the absence of FMRP, but have an equivalent cytoplasmic abundance [25]. Because in fragile $\mathrm{X}$ cells the distribution of FXR1P and FXR2P in polysomes is not changed, the change that we observed here must be specifically caused by the absence of FMRP. Comparing these two sets of mRNAs led us to identify 14 homologous mouse and human transcripts. Consistent with the abnormal dendritic spines found in both fragile $\mathrm{X}$ patients and Fmr1-knockout mouse, we have identified several FMRP mRNA ligands that are involved in synaptic functions, including Munc13, NAP-22, Rab6 interacting protein 1, SAPAP4, and Sec7-related guanine-nucleotide exchange factor (GEF) (Table 1). In addition, several mRNA ligands have been found important for axon
Table 1. Fragile $X$ mental retardation protein (FMRP) ligands related to synaptic plasticity and axon guidance

\begin{tabular}{|ll|}
\hline Synaptic plasticity & Axon guidance \\
\hline Fragile X mental retardation protein 1 & MAP1B \\
Munc13 & NAP-22 \\
NAP-22 & Semaphorin 3F \\
Rab6-interacting protein 1 & Inhibitor protein ID3 \\
SAPAP4 & \\
Sec7-related guanine-nucleotide exchange factor & \\
\hline
\end{tabular}

guidance, such as MAP1B, NAP-22, semaphorin3F and ID3 (Table 1). Another interesting observation from these microarray experiments is that in fragile $\mathrm{X}$ cells, the polyribosomal mRNA profiles show a much greater change compared with the expression profiles of whole cells. Indeed, $<0.05 \%$ of the RNAs were substantially changed when comparing wildtype with knockout whole-brain lysates, indicating that FMRP deficiency does not result in widespread mRNA changes at steady state and is consistent with the notion that FMRP is a translation regulator. Thus, the polyribosomal mRNA profile represents the molecular phenotype of fragile $\mathrm{X}$ cells more accurately and could be, potentially, used as a functional assay for drug development.

To identify the RNA sequence and structure that is required for FMRP-RNA interaction, two groups have independently found that an intramolecular G-QUARTET structure is essential for FMRP-RNA interaction [26,27]. Using the FMR1 mRNA, which has been previously shown to bind to FMRP in vitro, as a template Schaeffer et al. have reported that FMRP binds to the RGG-box coding region of FMR1 mRNA, which contains a purine-quartet motif [27]. Darnell et al. have taken a more systematic approach by using an in vitro selection of random RNA sequences (SELEX) that bind to FMRP [26]. They identified a consensus sequence with successive pairs of Guanosine residues, which forms an intramolecular G-quartet structure, and is crucial for binding. G quartets are hydrogenbonded structures formed from four guanosine residues in a square-planar array that are stabilized preferentially by $\mathrm{K}^{+}$and disrupted by the presence of $\mathrm{Li}^{+}$(Fig. 2a). Recognition of RNA by FMRP requires not only the G-quartet structure, but also specific sequences for the loop surrounding the G-quartet element because mutagenesis of nucleotides that are not involved in either the $\mathrm{G}$ quartet or the stem are able to markedly reduce FMRP binding. Based on the consensus sequence and structural requirement for FMRP binding, Darnell et al. have used a bio-informatic approach to search the UniGene database (www.ncbi.nih.gov/UniGene) and identified 71 potential FMRP binding sites. By making a series of truncated FMRP, they found that the RGG box, but not the KH domain, is responsible for the binding of specific mRNAs that contain this structure to FMRP. This is rather surprising given that the RGG box has long been considered as a nonspecific RNA-binding domain that unstacks adjacent nucleotide bases and unfolds RNA secondary structure to enable sequence-specific RNA binding. Examination of the mRNAs identified in the microarray analysis showed that nearly $70 \%$ of those transcripts that associate with FMRP in vivo and display 
(a)

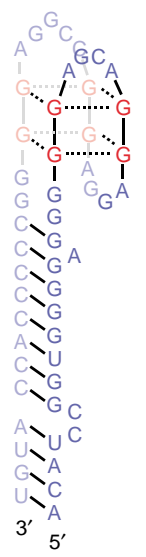

(b)

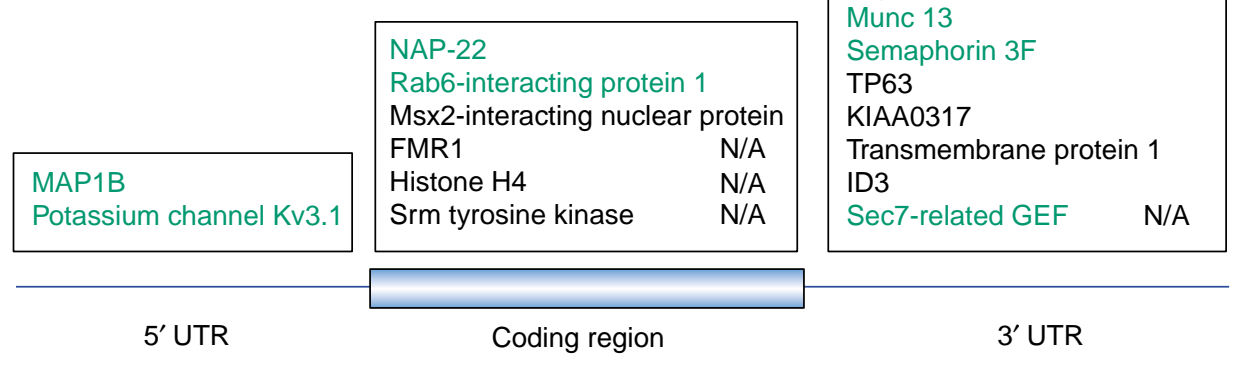

$\overline{\mathrm{TiBS}}$

Fig. 2. (a) Schematic representation of G-quartet and stem structure required for fragile $X$ mental retardation protein (FMRP)-mRNA interaction. The structure was generated based on the sequence of Sec7-related guanine-nucleotide exchange factor (GEF), one of the most enriched mRNAs in the immunoprecipitations from mouse brain. (b) Position of the G-quartet in the FMRP-mRNA ligands. The effects on polyribosomal association in fragile X patients are indicated (N/A, not available).

abnormal polyribosome profiles in the absence of FMRP contain a G-quartet structure [25,26]. Considering that $<4 \%$ of a random collection of $\mathrm{cDNAs}$ are predicted to be possible FMRP targets, this suggests that the G-quartet structure is physiologically relevant in fragile $\mathrm{X}$ syndrome.

Although it has been proposed that FMRP is involved in translational control, the exact role of FMRP in translation (as activator or suppressor) remains unclear. Several in vitro studies have shown that pre-incubation of mRNAs with FMRP leads to translation inhibition $[28,29]$. However, this effect showed little or no specificity of mRNA sequence. Using the immortal Fmr1-knockout cell line and transient transfections, it was shown that trapping of mRNA by FMRP into cytoplasmic granules can induce repression of translation [30]. The only in vivo data that might suggest the role of FMRP in translation has come from Drosophila studies [31]. In flies, the $d f m r 1$ protein was shown to repress expression of the futsch protein (microtubule-associated protein 1B ortholog) [31]. However, from these data we can not conclude that FMRP is a translational repressor because there is only a single gene encoding FMRP in Drosophila rather than the three genes that are present in mammals. Identification of the FMRP mRNA ligands and the RNA structure that is required for the FMRP-RNA interaction will help us address the in vivo role of FMRP in translation, although it appears to be more complex than previously thought. First, the microarray studies showed that the mRNAs associated with FMRP in vivo display different polyribosome profiles in the absence of FMRP; some are increased, whereas others are decreased [25]. Second, the G-quartet structure was found at different positions within the mRNA: within the $5^{\prime}$ UTR, coding region and $3^{\prime}$ UTR (Fig. 2b). There appears to be no correlation between the change in polyribosomes and the location of the G-quartet structure.

It has been proposed that post-translational modification of FMRP regulates its activity. However, the type of modification had not been identified until recently [32]. Now, it has been shown that both human FMRP and fly $d f m r 1$ protein are phosphorylated in vivo [33]. The phosphorylation site is conserved through evolution at Ser406 in $d f m r 1$ protein and Ser500 in human FMRP. In Drosophila, the homomer formation and RNA-binding activities of $d f m r 1$ protein are modulated by phosphorylation in vitro. In addition, Drosophila casein kinase II $(d C K I I)$ directly interacts with, and phosphorylates, $d f m r 1$ protein in vitro. The alteration of the conserved serine residue in both FMRP and $d f m r 1$ protein abolishes phosphorylation by CKII in vitro [33]. However, it will be important to determine which kinase phosphorylates FMRP and dfmr1 protein in vivo, and how the phosphorylation regulates FMRP activity in vivo.

Intriguingly, $d f m r 1$ was recently observed associated with RNA-induced silencing complex (RISC), a sequencespecific nuclease complex that mediates RNA interference (RNAi) in Drosophila [34,35]. RNAi is a conserved genesilencing response to double-stranded RNA (dsRNA). Silencing is initiated when dsRNA triggers are processed into small interfering RNAs (siRNAs); this is catalyzed by a group of related RNase III enzymes, now known as the Dicer family [36]. The siRNAs are then incorporated into the effector complex, RISC, which uses siRNA as a guide to select complementary mRNA substrates [36]. The same pathway has also been shown to process short hairpin RNAs that have been transcribed from endogenous genes, producing 21-nucleotide single-stranded microRNAs, putative translational regulators [37,38]. MicroRNAs can silence gene expression by repressing the translation of complementary mRNA transcripts [38]. But what is the physiological relevance of the association of $d f m r 1$ protein with RISC? It is unlikely that it affects $\mathrm{mRNA}$ degradation because microarray analysis has shown that very few mRNAs are substantially changed in either human patient cells or Fmr1-knockout mouse brain [25]. But it is possible that - similar to small temporal RNAs (stRNAs) lin-4 and let-7 in Caenorhabditis elegans - the translational regulation by FMRP is mediated through microRNAs [39]. FMRP could be targeted to its mRNA ligands as part of a RISC that is guided by FMRP itself or associated microRNAs. However, as Drosophila possesses only one gene from the fragile X-related gene family, it will be 
important to determine whether all three proteins (FMRP, FXR1P and FXR2P) or selective proteins are associated with RISC in mammals, as well to identify their associated microRNAs.

\section{Physiological consequences of FMRP deficiency in the brain}

To improve our understanding of the physiological functions of FMRP in the brain, both the Fmr1-knockout mouse and the recently developed $d f m r 1$-mutant flies have been studied $[3,9,15,31,40,41]$. Previous research has shown that the Fmr1-knockout mouse has subtle defects in behavior and spatial learning compared with wild-type mice [3]. Using various experimental paradigms, the Fmr1-knockout mouse has been reported to display increased sensitivity to audiogenic epileptic seizures, and greater response to low-intensity auditory stimuli [42-44]. In addition, abnormal responses to fear conditioning have been observed in some experiments but not in others, which might be because of strain differences [45-47]. Some of these manifestations could be correlated with the behavioral phenotype in fragile $\mathrm{X}$ patients. Overexpression of FMRP in Fmr1-knockout mice overcorrects this phenotype and produces opposing behavioral responses. The Fmr1-knockout mice have increased anxiety (i.e. they are hyperactive), whereas mice overexpressing FMRP display hypoactivity, suggesting that FMRP acts in a dose-dependent manner [48]. Moreover, increased rates of cerebral glucose metabolism have been observed in Fmr1-knockout mice; and the regions most affected are consistent with behavioral deficiencies and regions with highest FMRP expression [49]. Recently, Drosophila has been used as a model system to explore the function of FMRP. Adult $d f m r 1$-mutant flies exhibit arrhythmic circadian activity and impaired coordinated behavior, indicating potential deficits in locomotor functions [9,40,41]. $d$ fmr1-mutant males also display reduced courtship activity, which appears to result from their inability to maintain courtship interest [40]. All of these behavioral phenotypes can be rescued or even reversed (corrected) by overexpression of $d f m r 1$ [9,40,41]. This is consistent with the observation that regulation by FMRP is dosage-dependent in Fmr1-knockout mice. As Drosophila encode only a single gene from the fragile X-related gene family, it is not certain which of these phenotypes are related to fragile $\mathrm{X}$ syndrome in human, particularly considering that Fxr2-knockout mice have more severe behavioral phenotypes than the Fmr1-knockout mice. One way to address this question is to use mammalian Fmr1, Fxr 1 and Fxr2 to rescue the fly $d f m r 1$ mutant separately. Despite this, the establishment of the fragile X fly model should provide us with a powerful genetic model tool for conducting genetic screens for enhancers and suppressors, and to enable dissection of multiple genetic pathways that are regulated by $d f m r 1$ because the genetic pathways are commonly conserved across species during evolution.

How does the loss of FMRP lead to these behavioral phenotypes in fly, mouse and human?

The accumulating data suggest that FMRP regulates synaptic plasticity. The involvement of FMRP in synaptic plasticity was first indicated by the observation of abnormally long dendritic spines with increased density in fragile X patients, which is consistent with the idea that DENDRITIC SPINE DYSGENESIS is associated with human mental retardation [14,50]. Studies in both Fmr1-knockout mice and $d f m r 1$-mutant flies have confirmed that abnormal development of dendritic spines is associated with the loss of FMRP [15,31]. In the Fmr1-knockout mice, the dendritic spines are abnormal early in postnatal life [15]. Using the single-neuron labeling technique and twophoton microscopy, it has been found that the abnormalities of dendritic spines in the developing brain are most pronounced in the somatosensory cortex during the period of greatest synaptogenesis in that region. Interestingly, these anomalies appear to be transient, and subside largely by the end of the first postnatal month. Based on this strong developmental dependence of the abnormality in the intact brain, it has been suggested that FMRP might not function simply to maintain dendritic spines but, rather, to coordinate with normal activity. Additional supporting evidence includes increased expression of FMRP by afferent stimulation and elevation of local translation of FMRP in response to metabotropic glutamate receptor stimulation $[51,52]$. Several types of neuron have been examined in $d f m r 1$ mutant flies. In the neuromuscular junction (NMJ), synaptic terminals exhibit more growth and branching, and the number of SYNAPTIC BOUTONS is increased by $50 \%$. Conversely, overexpression of $d f m r 1$ in either pre- or post-synaptic compartments results in a decrease in the number of synaptic boutons [31]. The size of synaptic boutons also increases when $d f m r 1$ is overexpressed presynaptically. The $d f m r 1$ synaptic phenotypes in the NMJ mimic defects observed in mutants with altered levels of futsch, which is an mRNA ligand of FMRP in mammals. Interestingly, a dfmr1-futsch double mutant restores the $d f m r 1$ synaptic structural and functional defects, which is a surprising finding given the potentially large number of mRNA ligands of the mammalian FMRP protein [31]. In fly dorsal cluster (DC) neurons, both loss and gain of $d f m r 1$ function leads to the loss of neurite extension and irregular branching, as well as axon guidance defects. Lateral neurons (LNv), which control circadian rhythms, show variable defects in extension and guidance $[9,40]$. However, unlike the $\mathrm{NMJ}$, defects in LNv can not be rescued by the introduction of a futsch loss-of-function mutation, which might imply that $d f m r 1$ differentially regulates diverse targets in the brain $[9,40]$. Nonetheless, these data from different model systems strongly suggest that FMRP is involved in the regulation of synaptic plasticity.

\section{How does FMRP perform this role?}

Protein synthesis has long been considered a necessary and important component of synaptic plasticity, and different lines of evidence suggest that specificity of synaptic plasticity is established in part through local protein synthesis at individual synapses in dendrites [51,52]. Intriguingly, using electron microscopy, FMRP, as a translational regulator, has been detected in dendrites and dendritic spines, and also associated with translating polyribosomes in dendrites [12]. Thus, it has been 
suggested that the loss of FMRP will result in the misregulation of local protein synthesis of specific mRNAs, leading to altered synaptic plasticity. However, the physiological connection between the misregulated local translation of FMRP mRNA ligands and morphological defects of synapses in the absence of FMRP has remained elusive until recently. This missing link has now been identified as a form of protein-synthesis-dependent long-term depression (LTD) triggered by activation of metabotropic glutamate receptors (mGluR-LTD) [53]. There are two major types of protein-synthesis-dependent synaptic plasticity: long-term potentiation (LTP) and LTD. LTP is a long-term increase in synaptic strength in response to high-frequency stimulation, whereas LTD is a decrease in the strength of the same synapses after prolonged, low-frequency stimulation. There are two wellstudied forms of LTD, N-methyl-D-aspartate receptormediated LTD (NMDAR-LTD) and mGluR-LTD [54]. Although LTP is unaffected in the hippocampus of Fmr1knockout mice, mGluR-LTD, but not NMDAR-LTD, is significantly altered $[53,55]$. Using two distinct induction protocols (synaptic stimulation and induction by the highly specific group $1 \mathrm{mGluR}$ agonist DHPG), it was found that mGluR-LTD is significantly increased in the hippocampus of Fmr1-knockout mice. The role of FMRP in this form of synaptic plasticity is further indicated by the fact that FMRP is one of the proteins known to be synthesized in response to mGluR activation [52]. It has been proposed that FMRP regulates mGluR-LTD through local mRNA translation [53]. With the identification of FMRP mRNA ligands, it will be interesting to examine the role of those ligands in LTD expression, particularly those mRNAs located in dendrites, and also to study how FMRP regulates dendritic protein synthesis in vivo. However, if mGluR activation is the bridge between FMRP loss and spine dysgenesis, mGluR antagonists make an attractive therapeutic approach.

\section{Concluding remarks}

Recent advances in fragile $\mathrm{X}$ syndrome have provided new avenues to understand the molecular pathogenesis of this disease. Identification of FMRP mRNA ligands and the RNA structure required for the FMRP-RNA interaction will help to understand the role of FMRP in protein synthesis during neuronal development. The establishment of fly models will be very useful and important to dissect the physiological pathways regulated by FMRP using genetic approaches. Moreover, finding enhanced mGluR-LTD in Fmr1-knockout mice could open up new pharmalogical approaches for treating fragile X syndrome. By integrating biochemical, genetic, genomic and physiological approaches, the connection between molecules and neurobehaviors has been established, which is an important step towards understanding the molecular basis of fragile $\mathrm{X}$ syndrome and developing effective interventions for this disease.

\section{Acknowledgements}

We thank Stephanie Ceman, Yue Feng, Tracie Rosser and Daniela Zarnescu for critical reading of the article, and Janelle Clark for assistance. Supported by NIH grants R37 HD20521 and PO1 HD35576. P.J. is supported by Rett Syndrome Research Foundation.

\section{References}

1 Warren, S.T. and Sherman, S.L. (2001) The fragile X syndrome. In The Metabolic and Molecular Bases of Inherited Disease (Vol. 1) (Scriver, C.R. et al., eds), pp. 1257-1290, McGraw-Hill Companies

2 Ashley, C.T. et al. (1993) Human and murine FMR-1: alternative splicing and translational initiation downstream of the CGG-repeat. Nat. Genet. 4, 244-251

3 The Dutch-Belgian Fragile X Consortium. (1994) Fmr1 knockout mice: a model to study fragile $\mathrm{X}$ mental retardation. Cell 78, 23-33

4 Devys, D. et al. (1993) The FMR-1 protein is cytoplasmic, most abundant in neurons and appears normal in carriers of a fragile $\mathrm{X}$ premutation. Nat. Genet. 4, 335-340

5 Siomi, M.C. et al. (1995) FXR1, an autosomal homolog of the fragile X mental retardation gene. EMBO J. 14, 2401-2408

6 Zhang, Y. et al. (1995) The fragile X mental retardation syndrome protein interacts with novel homologs FXR1 and FXR2. EMBO J. 14, $5358-5366$

7 Feng, Y.et al. (1997) FMRP associates with polyribosomes as an mRNP, and the $\mathrm{I} 304 \mathrm{~N}$ mutation of severe fragile $\mathrm{X}$ syndrome abolishes this association. Mol. Cell 1, 109-118

8 Wan, L. et al. (2000) Characterization of dFMR1, a Drosophila melanogaster homolog of the fragile $\mathrm{X}$ mental retardation protein. Mol. Cell. Biol. 20, 8536-8547

9 Morales, J. et al. (2002) Drosophila fragile X protein, DFXR, regulates neuronal morphology and function in the brain. Neuron 34, 961-972

10 Ashley, C.T. Jr et al. (1993) FMR1 protein: conserved RNP family domains and selective RNA binding. Science 262, 563-565

11 Eberhart, D.E. et al. (1996) The fragile X mental retardation protein is a ribonucleoprotein containing both nuclear localization and nuclear export signals. Hum. Mol. Genet. 5, 1083-1091

12 Feng, Y. et al. (1997) Fragile X mental retardation protein: nucleocytoplasmic shuttling and association with somatodendritic ribosomes. J. Neurosci. 17, 1539-1547

13 Comery, T.A. et al. (1997) Abnormal dendritic spines in fragile X knockout mice: maturation and pruning deficits. Proc. Natl. Acad. Sci. U. S. A. 94, 5401-5404

14 Hinton, V.J. et al. (1991) Analysis of neocortex in three males with the fragile X syndrome. Am. J. Med. Genet. 41, 289-294

15 Nimchinsky, E.A. et al. (2001) Abnormal development of dendritic spines in FMR1 knock-out mice. J. Neurosci. 21, 5139-5146

16 Ceman, S. et al. (1999) Isolation of an FMRP-associated messenger ribonucleoprotein particle and identification of nucleolin and the fragile X-related proteins as components of the complex. Mol. Cell. Biol. 19, 7925-7932

17 Ceman, S. et al. (2000) Identification of mouse YB1/p50 as a component of the FMRP-associated mRNP particle. Biochem. Biophys. Res. Commun. 279, 904-908

18 Ohashi, S. et al. (2002) Identification of mRNA/protein (mRNP) complexes containing Puralpha, mStaufen, fragile $\mathrm{X}$ protein, and myosin $\mathrm{Va}$ and their association with rough endoplasmic reticulum equipped with a kinesin motor. J. Biol. Chem. 277, 37804-37810

$19 \mathrm{Li}$, Y. et al. (2001) Pur alpha protein implicated in dendritic RNA transport interacts with ribosomes in neuronal cytoplasm. Biol. Pharm. Bull. 24, 231-235

20 Kiebler, M.A. et al. (1999) The mammalian staufen protein localizes to the somatodendritic domain of cultured hippocampal neurons: implications for its involvement in mRNA transport. J. Neurosci. 19, 288-297

21 Duchaine, T.F. et al. (2002) Staufen2 isoforms localize to the somatodendritic domain of neurons and interact with different organelles. J. Cell Sci. 115, 3285-3295

22 De Diego Otero, Y. et al. (2002) Transport of fragile X mental retardation protein via granules in neurites of $\mathrm{PC} 12$ cells. Mol. Cell. Biol. 22, 8332-8341

23 Bardoni, B. et al. (1999) A novel RNA-binding nuclear protein that interacts with the fragile X mental retardation (FMR1) protein. Hum. Mol. Genet. 8, 2557-2566

24 Schenck, A. et al. (2001) A highly conserved protein family interacting 
with the fragile $\mathrm{X}$ mental retardation protein (FMRP) and displaying selective interactions with FMRP-related proteins FXR1P and FXR2P Proc. Natl. Acad. Sci. U. S. A. 98, 8844-8849

25 Brown, V. et al. (2001) Microarray identification of FMRP-associated brain mRNAs and altered mRNA translational profiles in fragile $\mathrm{X}$ syndrome. Cell 107, 477-487

26 Darnell, J.C. et al. (2001) Fragile X mental retardation protein targets G quartet mRNAs important for neuronal function. Cell 107, 489-499

27 Schaeffer, C. et al. (2001) The fragile X mental retardation protein binds specifically to its mRNA via a purine quartet motif. EMBO J. 20 , 4803-4813

28 Laggerbauer, B. et al. (2001) Evidence that fragile $\mathrm{X}$ mental retardation protein is a negative regulator of translation. Hum. Mol. Genet. 10, 329-338

$29 \mathrm{Li}$, Z. et al. (2001) The fragile X mental retardation protein inhibits translation via interacting with mRNA. Nucleic Acids Res. 29 2276-2283

30 Mazroui, R. et al. (2002) Trapping of messenger RNA by fragile X mental retardation protein into cytoplasmic granules induces translation repression. Hum. Mol. Genet. 11, 3007-3017

31 Zhang, Y.Q. et al. (2001) Drosophila fragile X-related gene regulates the MAP1B homolog Futsch to control synaptic structure and function. Cell 107, 591-603

32 Ceman, S. et al. (2001) A single amino acid substitution in the FMR protein $(\mathrm{I} 304 \mathrm{~N})$ results in reduced phosphorylation and an altered conformation. Am. J. Hum. Genet. 69, 348

33 Siomi, M.C. et al. (2002) Casein kinase II phosphorylates the fragile X mental retardation protein and modulates its biological properties. Mol. Cell. Biol. 22, 8438-8447

34 Caudy, A.A. et al. (2002) Fragile X-related protein and VIG associate with the RNA interference machinery. Genes Dev. 16, 2491-2496

35 Ishizuka, A. et al. (2002) A Drosophila fragile X protein interacts with components of RNAi and ribosomal proteins. Genes Dev. 16, 2497-2508

36 Hammond, S.M. et al. (2001) Post-transcriptional gene silencing by double-stranded RNA. Nat. Rev. Genet. 2, 110-119

37 Eddy, S.R. (2001) Non-coding RNA genes and the modern RNA world. Nat. Rev. Genet. 2, 919-929

38 Gottesman, S. (2002) Stealth regulation: biological circuits with small RNA switches. Genes Dev. 16, 2829-2842

39 Pasquinelli, A.E. and Ruvkun, G. (2002) Control of developmental timing by micrornas and their targets. Annu. Rev. Cell Dev. Biol. 18, $495-513$

40 Dockendorff, T.C. et al. (2002) Drosophila lacking $d f m r 1$ activity show defects in circadian output and fail to maintain courtship interest. Neuron 34, 973-984

41 Inoue, S. et al. (2002) A role for the Drosophila fragile x-related gene in circadian output. Curr. Biol. 12, 1331-1335

42 Musumeci, S.A. et al. (2000) Audiogenic seizures susceptibility in transgenic mice with fragile X syndrome. Epilepsia 41, 19-23

43 Chen, L. and Toth, M. (2001) Fragile X mice develop sensory hyperreactivity to auditory stimuli. Neuroscience 103, 1043-1050

44 Nielsen, D.M. et al. (2002) Alterations in the auditory startle response in Fmr 1 targeted mutant mouse models of fragile X syndrome. Brain Res. 927, 8-17

45 Paradee, W. et al. (1999) Fragile X mouse: strain effects of knockout phenotype and evidence suggesting deficient amygdala function. Neuroscience 94, 185-192

46 Van Dam, D. et al. (2000) Spatial learning, contextual fear conditioning and conditioned emotional response in Fmr1 knockout mice. Behav. Brain Res. 117, 127-136

47 Dobkin, C. et al. (2000) Fmr1 knockout mouse has a distinctive strainspecific learning impairment. Neuroscience 100, 423-429

48 Peier, A.M. et al. (2000) (Over)correction of FMR1 deficiency with YAC transgenics: behavioral and physical features. Hum. Mol. Genet. 9, 1145-1159

49 Qin, M. et al. (2002) Increased rates of cerebral glucose metabolism in a mouse model of fragile X mental retardation. Proc. Natl. Acad. Sci. U. S. A. 99, 15758-15763

50 Purpura, D.P. (1974) Dendritic spine 'dysgenesis' and mental retardation. Science 186, 1126-1128

51 Greenough, W.T. et al. (2001) Synaptic regulation of protein synthesis and the fragile X protein. Proc. Natl. Acad. Sci. U. S. A. $98,7101-7106$

52 Weiler, I.J. and Greenough, W.T. (1999) Synaptic synthesis of the fragile X protein: possible involvement in synapse maturation and elimination. Am. J. Med. Genet. 83, 248-252

53 Huber, K.M. et al. (2002) Altered synaptic plasticity in a mouse model of fragile X mental retardation. Proc. Natl. Acad. Sci. U. S. A. 99, 7746-7750

54 Huber, K.M. et al. (2000) Role for rapid dendritic protein synthesis in hippocampal mGluR- dependent long-term depression. Science 288, 1254-1257

55 Godfraind, J.M. et al. (1996) Long-term potentiation in the hippocampus of fragile X knockout mice. Am. J. Med. Genet. 64, 246-251

\section{Managing your references and BioMedNet Reviews}

Did you know that you can now download selected search results from BioMedNet Reviews directly into your chosen reference-managing software? After performing a search, simply click to select the articles you are interested in, choose the format required

(e.g. EndNote 3.1) and the bibliographic details, abstract and link to the full-text will download into your desktop reference manager database. 